\title{
Sérovars de Salmonella enterica associés à des bactériémies au Canada, 2006 à 2019
}

\author{
Sandeep Tamber ${ }^{1 *}$, Brendan Dougherty ${ }^{2}$, Kimberly Nguy ${ }^{2}$
}

\section{Résumé}

Contexte : Les membres du genre bactérien Salmonella sont à l'origine de la salmonellose, une maladie dont les manifestations cliniques varient d'une gastro-entérite autolimitée à une bactériémie plus grave, une défaillance organique et une septicémie. Le genre se compose de plus de 2600 variantes sérologiques (sérovars). On a remarqué des différences importantes dans la pathogenèse des sérovars de Salmonella.

Objectif : Le but de cette étude était de déterminer quels sérovars de Salmonella étaient plus susceptibles d'être associés à des bactériémies au Canada.

Méthodes : Pour chaque sérovar, on a extrait les informations sur le nombre total d'infections à Salmonella et d'isolats sanguins signalés au Programme national de surveillance des maladies entériques de 2006 à 2019. Le risque (proportion) et la probabilité (cote) de bactériémie ont été calculés pour tous les sérovars.

Résultats : Sur les 96082 cas de salmonellose signalés au Programme national de surveillance des maladies entériques pendant la période d'étude de 14 ans, 4,4 \% (IC $95 \%$ : 4,3 \%-4,6\%) étaient bactériémiques. Vingt sérovars de Salmonella non typhique ont été associés à des taux plus faibles de bactériémie par rapport à tous les sérovars de Salmonella non typhique, et 19 sérovars de Salmonella non typhique ont été identifiés comme ayant des taux plus élevés, Heidelberg, Oranienburg, Schwarzengrund, Virchow, Panama et Poona comptant parmi les 25 sérovars les plus fréquemment signalés au Canada pendant la période d'étude.

Conclusion : L'identification des sérovars associés aux bactériémies à Salmonella au Canada est un premier pas vers la compréhension des différences dans la pathogenèse et la présentation de la maladie.
Cette oeuvre est mise à la disposition selon les termes de la licence internationale Creative Commons Attribution 4.0

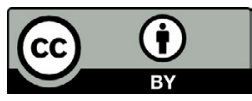

Affiliations

1 Direction des aliments, Santé Canada, Ottawa, ON

${ }^{2}$ Centre des maladies infectieuses d'origine alimentaire, environnementale et zoonotique, Agence de la santé publique du Canada, Ottawa, ON

\section{*Correspondance :}

sandeep.tamber@canada.ca

Citation proposée : Tamber S, Dougherty B, Nguy K. Sérovars de Salmonella enterica associés à des bactériémies au Canada, 2006 à 2019. Relevé des maladies transmissibles au Canada 2021;47(5/6):284-93.

https://doi.org/10.14745/ccdr.v47i56a03f

Mots-clés : typhique, salmonellose non typhique, Programme national de surveillance des maladies entériques, PNSME

\section{Introduction}

Les bactéries de Salmonella sont une cause importante de maladie humaine et comptent une variété de présentations cliniques (1). Le genre comprend deux espèces, six sous-espèces et plus de 2600 variantes sérologiques (sérovars). Bien que toutes les espèces et sous-espèces aient été rapportées comme pouvant causer des maladies, la plupart des infections humaines sont causées par S. enterica spp. enterica (sous-espèce I) (2). La sous-espèce I comprend des sérovars typhiques et non typhiques. Les sérovars typhiques provoquent la fièvre typhique (entérique), une infection invasive grave qui peut entraîner une défaillance de plusieurs organes, une bactériémie et une septicémie (3). La salmonellose non typhique se traduit principalement par une gastro-entérite aiguë autolimitée, bien que des infections plus graves puissent survenir (3). Au Canada, la majorité des cas de salmonellose signalés annuellement, y compris environ 925 hospitalisations et 17 décès, sont dus à la Salmonella non typhique (4).

L'une des caractéristiques de Salmonella est la présence de gènes de virulence codés sur des sections du génome appelées îlots de pathogénicité de Salmonella. Les 2659 sérovars de Salmonella possèdent tous des îlots de pathogénicité de Salmonella et codent donc la capacité à provoquer des infections (5). Néanmoins, seule une petite proportion de 
sérovars est responsable de la majorité des infections humaines signalées, plus de $80 \%$ des cas de salmonellose signalés étant attribués à 20 sérovars alors que plus de 200 sérovars sont signalés chaque année au Canada (6). Bien que certains sérovars fréquemment signalés sont associés à des risques d'exposition plus importants parce qu'ils sont plus répandus dans l'environnement, la présence de plusieurs sérovars très répandus, mais rarement signalés suggère des différences de virulence entre les sérovars (7). Cette hypothèse est soutenue par des études sur la pathogenèse des sérovars typhiques et des sérovars de Salmonella non typhique tels que Choleraesuis, Dublin, Typhimurium et Enteritidis $(1,7)$. La pathogenèse de la plupart des sérovars de la Salmonella non typhique est cependant peu étudiée et donc mal comprise.

L'examen des taux de bactériémie à Salmonella chez les humains est un moyen de comparer la virulence relative des sérovars. Mondialement, environ $2 \%$ à $8 \%$ des infections signalées de la salmonellose non typhique conduisent à une bactériémie, et dans certains cas, la bactériémie n'est pas précédée d'une gastro-entérite (8-11). Les patients atteints de bactériémie sont plus susceptibles de connaître des issues graves, notamment I'hospitalisation et le décès, s'ils ne sont pas traités rapidement par des antibiotiques $(12,13)$. Par conséquent, l'identification précoce des patients à haut risque peut améliorer le pronostic des bactériémies à Salmonella $(9,14)$. Les facteurs de risque humains pour la dissémination bactérienne à partir de l'intestin comprennent une immunité compromise, des conditions médicales sous-jacentes et des âges extrêmes. Les facteurs de virulence bactérienne impliqués dans ce processus comprennent des protéines d'adhésion, des protéines d'évasion immunitaire et d'autres effecteurs sécrétés $(3,10,15,16)$.

L'objectif de cette étude était de comparer les taux de bactériémie de différentes sous-espèces et sérovars de Salmonella afin de mieux comprendre les différences de virulence. L'identification des sérovars de Salmonella qui sont plus ou moins susceptibles d'être associés à une bactériémie est une étape clé vers le développement de meilleurs prédicteurs de la bactériémie à Salmonella et, à terme, de meilleures normes de soins.

\section{Méthodes}

\section{Sources de données}

L'équipe de recherche a obtenu des données du Programme national de surveillance des maladies entériques, qui est administré par l'Agence de la santé publique du Canada (6). Les données sont un résumé des données de laboratoire soumises chaque semaine par les laboratoires de microbiologie de la santé publique provinciaux et territoriaux au Programme national de surveillance des maladies entériques. Aux fins de cette analyse, nous avons extrait les données de 2006 à 2019, y compris le nombre d'infections à Salmonella par sous-espèce, sérovar et source de spécimen pour les isolations extra-intestinales. Chaque isolation signalée représente un cas clinique. Les isolats sanguins étant récupérés dans le sang et pris comme approximation du nombre de cas de bactériémie causés par la Salmonella (bactériémie à Salmonella). Les isolats totaux sont ceux qui ont été récupéré à partir de l'ensemble des sources d'échantillons prélevées.

\section{Analyse des données}

Les informations sur la fréquence de signalement et la distribution des sous-espèces et des sérovars de Salmonella ont été mises sous forme de tableaux à l'aide de Microsoft Excel 2016 et visualisées à l'aide de GraphPad Prism 8.0.2.

Nous avons calculé les taux de bactériémie à Salmonella au fil du temps en divisant le nombre $d$ 'isolats sanguins déclarés chaque année par le nombre total de signalements de Salmonella pour cette année. Le test de tendance du chi carré/Cochran-Armitage a été utilisé pour déterminer s'il existait une tendance linéaire dans les taux de bactériémie au fil du temps. Cette analyse temporelle a été menée au niveau du genre ainsi qu'au niveau des sérovars de Salmonella non typhique individuels.

Les proportions de bactériémie (PB) des différents sérovars ou groupes de Salmonella ont été calculées en divisant le nombre d'isolats sanguins d'un sérovar/groupe donné par le nombre total d'isolats du même sérovar/groupe pour toutes les années combinées. Les intervalles de confiance (IC) à $95 \%$ pour la proportion de bactériémie ont été calculés à l'aide de la formule

$$
\mathrm{BP} \pm \frac{1.96 \sqrt{\mathrm{BP}(1-\mathrm{BP})}}{\mathrm{n}}
$$

suivante :

Pour certaines analyses, nous avons regroupé les sérovars selon la désignation de leur sous-espèce (I-VI) et la présentation de la maladie (typhique ou non typhique). Les taux de bactériémie significativement plus élevés ou plus faibles dans les sérovars ou groupes de Salmonella individuels ont été évalués à l'aide du test exact de Fisher. Pour identifier les sous-espèces et les sérovars présentant des taux plus élevés d'isolations sanguines, nous avons comparé les groupes suivants : la sous-espèce I par rapport à toutes les autres sous-espèces (II, IIla, IIIb, IV et II-VI); les sérovars typhiques par rapport aux sérovars de Salmonella non typhique; et un seul sérovar de Salmonella non typhique par rapport à tous les sérovars de Salmonella non typhique.

Les rapports de cotes ont été calculés en comparant la probabilité qu'un sérovar donné infecte le sang(a/b) à la probabilité que toute souche de Salmonella non typhique infecte le sang $(c / d)$; où $a$ et $b$ sont les nombres respectifs $d$ 'isolats sanguins et non sanguins pour chaque sérovar et $c$ et $d$ sont les nombres correspondants pour toutes les souches de Salmonella non typhique. Nous avons utilisé la formule suivante pour calculer I'IC à $95 \%$ du rapport des cotes :

$$
e\left(\left[\ln (O R) \pm 1.96 \sqrt{\left(\frac{1}{a}+\frac{1}{b}+\frac{1}{d}\right]}\right)\right.
$$


Nous avons effectué tous les calculs et tests statistiques en utilisant Microsoft Excel 2016 et GraphPad Prism 8.0.2. Une valeur-p inférieure à 0,05 a été considérée comme significative pour tous les tests statistiques.

\section{Résultats}

De 2006 à 2019, 96082 isolations de Salmonella ont été signalées au Programme national de surveillance des maladies entériques. Au cours de cette période, 4252 isolations du système sanguin ont été signalées, soit un taux global de bactériémie de 4,4\% (IC $95 \%$ : $4.3 \%-4.6 \%$ ). L'étude des taux annuels de bactériémies a montré une tendance à la hausse sur la période de 14 ans, avec un taux d'augmentation annuel moyen de $0,2 \%$ (IC $95 \%$ : $-0,4 \%$ à $0,7 \%$ ) et une fourchette de $2,7 \%$ en 2007 (IC 95 \% : 2,4 \%-3,2 \%) à 5,9\% (IC $95 \%$ : 5,4 \%-6,5\%) en $2017(P<0,0001$, figure 1 et figure 2$)$.

Figure 1 : Nombre total de signalements et d'isolations sanguines de bactériémies à Salmonella signalées au PNSME, 2006 à 2019a

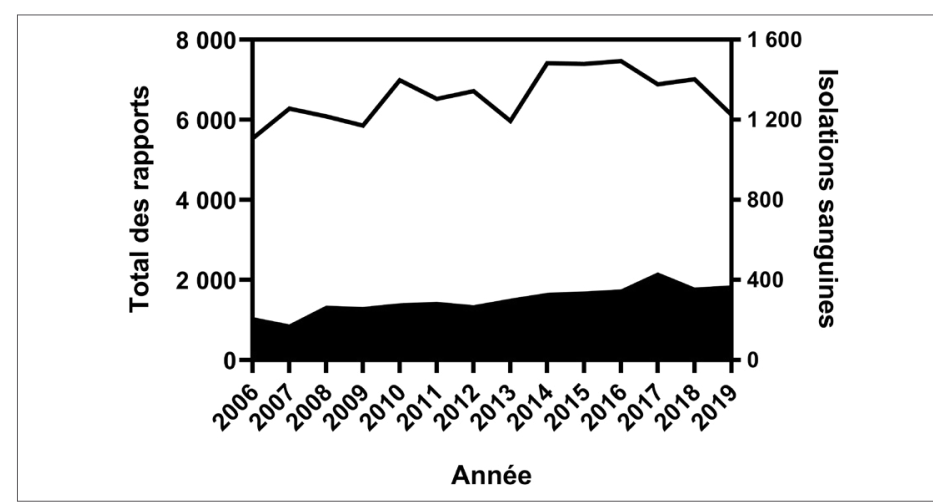

Abréviation : PNSME, Programme national de surveillance des maladies entériques a Isolations totales (ligne) et sanguines (forme pleine) rapportées au PNSME

Sur les 96082 isolats de Salmonella signalés, 95385 (99,3\%) ont été typés au niveau du sérovar, $258(0,3 \%)$ au niveau de la sous-espèce et $439(0,5 \%)$ au niveau du genre. Le groupe du genre a été exclu de l'analyse ultérieure. Parmi les 4252 isolats sanguins, sept ont été exclus de l'analyse parce qu'ils n'ont été typés qu'au niveau du genre.
Figure 2 : Évolution dans le temps des proportions de bactériémies à Salmonella, d'après les rapports adressés au PNSME, 2006 à 2019a

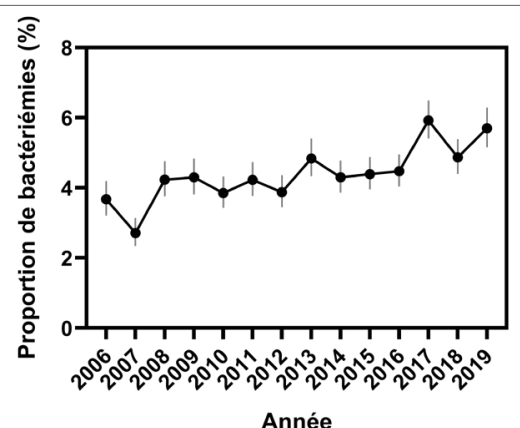

Abréviation : PNSME, Programme national de surveillance des maladies entériques a Les lignes verticales indiquent les intervalles de confiance à $95 \%$ Remarque : $P<0,0001$

Le tableau 1 présente la distribution des sous-espèces des isolats typés qui ont été rapportés. II n'y a eu que deux signalements de $S$. bongori pendant la période de référence; tous deux ont été isolés à partir de selles. Les autres isolats étaient des $S$. enterica, 99,3\% des isolats totaux et 99,5\% des isolats sanguins étant typés comme étant de la sous-espèce I.

Le taux de bactériémie de la sous-espèce I était de 4,4\% (IC $95 \%$ : $4.3 \%-4.6 \%$ ). À l'exception de la sous-espèce IIIb, ce taux ne différait pas significativement de ceux des autres sous-espèces, même lorsqu'elles étaient analysées en tant que groupe agrégé pour réduire l'imprécision associée au faible nombre d'isolats ( $P=0,0728$, figure 3 ). Le taux de bactériémie de la sous-espèce IIIb, 1,4\% (IC $95 \%$ : 0.5 \%-3,5\%), était significativement plus faible que celle de la sous-espèce I $(P=0,0087)$.

Parmi les isolats de la sous-espèce I, il y avait 3678 isolations totales de souches typhiques (2 350 Typhi, 1198 Paratyphi A, 124 Paratyphi B et 6 Paratyphi C) et 1295 isolations sanguines (794 Typhi, 485 Paratyphi A, 16 Paratyphi B et 0 Paratyphi C). Le taux de bactériémie des souches typhiques était de $35,2 \%$ (IC $95 \%$ : $33.7 \%-36,8 \%$ ), significativement plus élevé que le taux calculé pour les souches non typhiques $(3,2 \%$, IC $95 \%: 3.1 \%-3,3 \%, P<0,0001$, figure 4$)$.

Tableau 1 : Fréquence des espèces et sous-espèces de Salmonella signalées au PNSME, 2006 à 2019

\begin{tabular}{|c|c|c|c|c|c|}
\hline \multirow{2}{*}{ Espèce } & \multirow{2}{*}{ Sous-espèces } & \multicolumn{2}{|c|}{ Total des rapports } & \multicolumn{2}{|c|}{ Rapports sanguins } \\
\hline & & Nombre de sérovars & Nombre d'isolats & Nombre de sérovars & Nombre d'isolats \\
\hline \multirow{6}{*}{ Salmonella enterica } & enterica (I) & 574 & 94972 & 139 & 4218 \\
\hline & salamae (II) & 40 & 80 & 2 & 2 \\
\hline & arizoniae (IIla) & 19 & 104 & 4 & 5 \\
\hline & diarizonae (IIlb) & 95 & 285 & 4 & 4 \\
\hline & houtenae (IV) & 24 & 199 & 4 & 9 \\
\hline & indica $(\mathrm{VI})$ & 1 & 1 & 0 & 0 \\
\hline Salmonella bongori & s.o. & 1 & 1 & 0 & 0 \\
\hline
\end{tabular}

Abréviations : PNSME, Programme national de surveillance des maladies entériques; s.o., sans objet 
Figure 3 : Comparaison des proportions de bactériémies des sous-espèces de Salmonella enterica, sur la base des rapports au PNSME, 2006 à 2019a,b,c

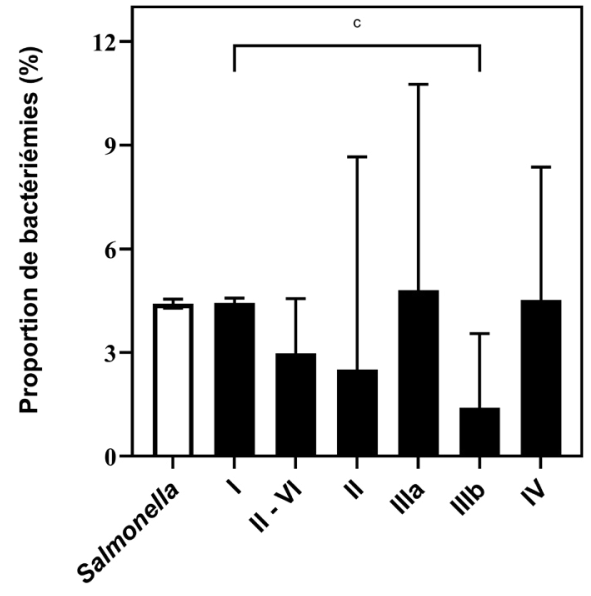

Groupe

Abréviation : PNSME, Programme national de surveillance des maladies entériques

a La barre blanche représente la proportion globale de bactériémies du genre Salmonella Les lignes verticales indiquent les intervalles de confiance à $95 \%$

c $P=0,0087$

Figure 4 : Comparaison des proportions de bactériémies dues à des souches typhiques et non typhiques des sous-espèces de Salmonella enterica, d'après les rapports transmis au PNSME, 2006 à 2019a,b,c

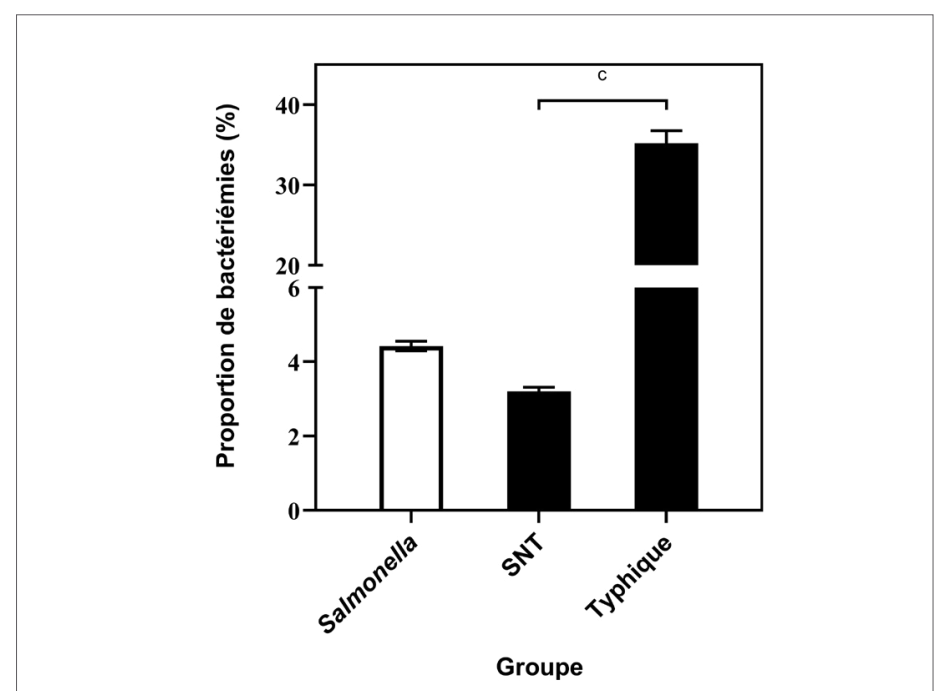

Abréviations : PNSME, Programme national de surveillance des maladies entériques; SNT, Salmonella non typhique

a La barre blanche représente la proportion globale de bactériémies du genre Salmonella ${ }^{b}$ Les lignes verticales indiquent les intervalles de confiance à $95 \%$ $P<0,0001$

Sur les 570 sérovars de la Salmonella non typhique signalés au Programme national de surveillance des maladies entériques de 2006 à 2019, 136 ont été associés à des bactériémies.

Enteritidis était le plus fréquemment associé à la bactériémie, avec plus de 1000 rapports. Avec Heidelberg, ces deux sérovars représentaient $60 \%$ des rapports sanguins (figure 5 ).
Typhimurium, Dublin et Oranienburg étaient, respectivement, les troisième, quatrième et cinquième sérovars les plus fréquemment signalés dans le sang (figure 5 ).

Figure 5 : Distribution de fréquence des cinq principaux sérovars non typhiques de Salmonella enterica isolés dans le sang, signalés au PNSME, 2006 à 2019a

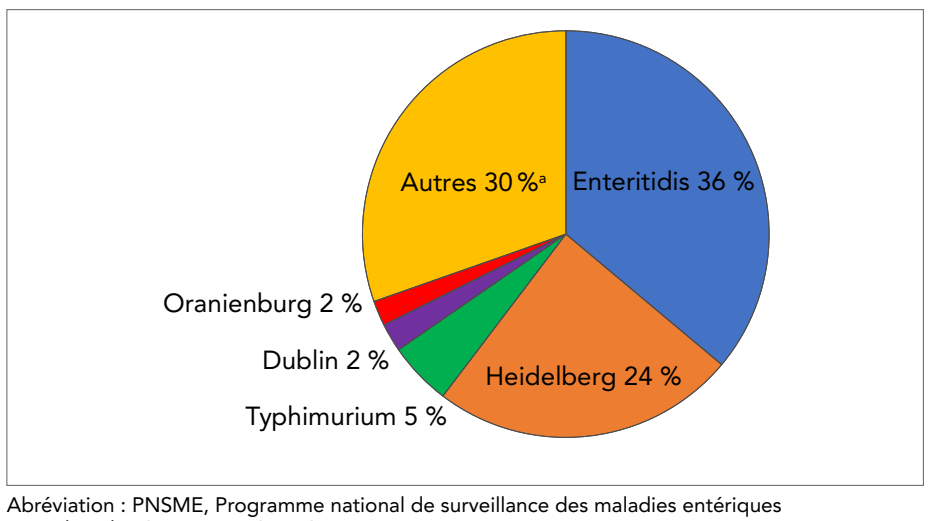

a Nombre de sérovars représentés par « Autres » $=131$

Soixante-trois sérovars n'ont été associés qu'à un seul rapport sanguin au cours de la période de signalement de 14 ans. À titre de comparaison, les deux principaux sérovars de la Salmonella non typhique, toutes sources de spécimens confondues, étaient Enteritidis et Typhimurium, représentant $50 \%$ du total des rapports. Les sérovars Heidelberg, I 4, [5],12:i:- et Infantis complètent le top 5 (figure 6).

Figure 6 : Distribution de fréquence des cinq principales Salmonella enterica non typhiques isolées de toutes les sources d'échantillons déclarées au PNSME, 2006 à 2019a

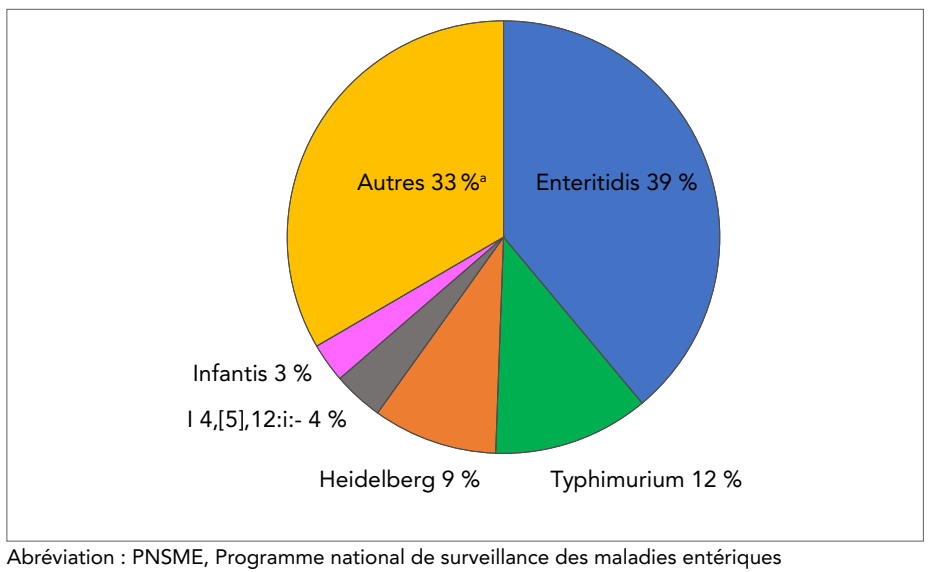

Nombre de sérovars représentés par « Autres » $=565$

Les proportions de bactériémie pour les sérovars individuels de la Salmonella non typhique associés à au moins 10 infections sont énumérées dans le tableau 2 par ordre décroissant de fréquence. 
Tableau 2 : Proportions de bactériémies des sérovars de Salmonella non typhiques ayant fait l'objet d'au moins 10 signalements au PNSME, 2006 à 2019

\begin{tabular}{|c|c|c|c|c|c|c|c|}
\hline Serovar ${ }^{a}$ & $\begin{array}{l}\text { Nombre } \\
\text { d'isolats } \\
\text { sanguins }\end{array}$ & $\begin{array}{l}\text { Nombre total } \\
\text { d'isolats }\end{array}$ & $\begin{array}{c}\text { Taux de } \\
\text { bactériémie } \\
(\%)^{b}\end{array}$ & IC À $95 \%(\%)$ & valeur-p ${ }^{c}$ & $\begin{array}{l}\text { Rapport des } \\
\text { cotes }^{d}\end{array}$ & IC À 95 \% \\
\hline SNT & 2917 & 91088 & 3,2 & $3,1-3,3$ & s.o. & s.o. & s.o. \\
\hline Enteritidise & $1053^{\circ}$ & $35459^{e}$ & $3,0^{\mathrm{e}}$ & $2,8-3,2^{e}$ & $0,0326^{e}$ & $0,9^{e}$ & $0,9-1,0$ e \\
\hline Typhimuriume & $147^{e}$ & $10617^{e}$ & $1,4^{\mathrm{e}}$ & $1,2-1,6^{e}$ & $<0,0001^{\mathrm{e}}$ & $0,4^{e}$ & $0,4-0,5^{\mathrm{e}}$ \\
\hline Heidelberg ${ }^{f}$ & $707^{f}$ & $8482^{f}$ & $8,3^{f}$ & $7,8-8,9^{f}$ & $<0,0001^{f}$ & $2,7^{f}$ & $2,5-3,0^{f}$ \\
\hline | 4,[5],12:i:-e & $52^{e}$ & $3430^{\circ}$ & $1,5^{\mathrm{e}}$ & $1,2-2,0^{e}$ & $<0,0001^{\mathrm{e}}$ & $0,5^{e}$ & $0,4-0,6^{\mathrm{e}}$ \\
\hline Infantise & $28^{e}$ & $2672^{\mathrm{e}}$ & $1,0^{\mathrm{e}}$ & $0,7-1,5^{e}$ & $<0,0001^{e}$ & $0,3^{e}$ & $0,2-0,5^{\mathrm{e}}$ \\
\hline Thompsone & $46^{e}$ & $2582^{e}$ & $1,8^{\mathrm{e}}$ & $1,3-2,4^{e}$ & $<0,0001^{\mathrm{e}}$ & $0,6^{\mathrm{e}}$ & $0,4-0,7 \mathrm{e}$ \\
\hline Newport ${ }^{\mathrm{e}}$ & $38^{e}$ & $2474^{e}$ & $1,5^{\mathrm{e}}$ & $1,1-2,1^{\mathrm{e}}$ & $<0,0001^{e}$ & $0,5^{e}$ & $0,3-0,7 \mathrm{e}$ \\
\hline Saintpaul & 47 & $1466^{e}$ & 3,2 & $2,4-4,2$ & 0,9405 & 1,0 & $0,7-1,3$ \\
\hline Javiana & 45 & $1384^{e}$ & 3,3 & $2,4-4,3$ & 0,8778 & 1,0 & $0,8-1,4$ \\
\hline Agona $^{e}$ & $15^{\mathrm{e}}$ & $1255^{e}$ & $1,2^{\mathrm{e}}$ & $0,7-2,0^{e}$ & $<0,0001^{\mathrm{e}}$ & $0,4^{e}$ & $0,2-0,6^{\mathrm{e}}$ \\
\hline Braenderupe & $6^{e}$ & $1208^{e}$ & $0,5^{\mathrm{e}}$ & $0,2-1,1^{e}$ & $<0,0001^{\mathrm{e}}$ & $0,2^{e}$ & $0,1-0,3^{e}$ \\
\hline Hadare & $13^{e}$ & $1077^{\mathrm{e}}$ & $1,2^{\mathrm{e}}$ & $0,7-2,0^{\circ}$ & $<0,0001^{\mathrm{e}}$ & $0,4^{e}$ & $0,2-0,6^{\mathrm{e}}$ \\
\hline Oranienburg ${ }^{f}$ & $58^{f}$ & $1063^{f}$ & $5,5^{f}$ & $4,2-7,0^{f}$ & $0,0002^{f}$ & $1,7^{f}$ & $1,3-2,3^{f}$ \\
\hline Stanleye & $19^{\mathrm{e}}$ & $981^{e}$ & $1,9^{e}$ & $1,2-3,0^{\circ}$ & $0,0221^{\mathrm{e}}$ & $0,6^{\mathrm{e}}$ & $0,4-0,9 \mathrm{e}$ \\
\hline Muenchene & $11^{\mathrm{e}}$ & $884^{e}$ & $1,2^{\mathrm{e}}$ & $0,7-2,2^{e}$ & $0,0003^{e}$ & $0,4^{e}$ & $0,2-0,7 \mathrm{e}$ \\
\hline | 4,[5],12:b:- & 24 & 880 & 2,7 & $1,8-4,0$ & 0,4998 & 0,9 & $0,6-1,3$ \\
\hline Paratyphi B var. Java ${ }^{f}$ & $41^{f}$ & $874^{f}$ & $4,7^{f}$ & $3,5-6,3^{f}$ & $0,0201^{f}$ & $1,5^{f}$ & $1,1-2,0^{f}$ \\
\hline Montevideo & 12 & 544 & 2,2 & $1,3-3,8$ & 0,2207 & 0,7 & $0,4-1,2$ \\
\hline Mbandaka $^{e}$ & $6^{\mathrm{e}}$ & $474^{e}$ & $1,3^{\mathrm{e}}$ & $0,6-2,7^{e}$ & $0,0122^{e}$ & $0,4^{e}$ & $0,2-0,9^{\mathrm{e}}$ \\
\hline Virchow ${ }^{f}$ & $28^{f}$ & $425^{f}$ & $6,6^{4}$ & $4,6-9,4^{f}$ & $0,0004^{f}$ & $2,1^{f}$ & $1,5-3,1^{f}$ \\
\hline Schwarzengrund ${ }^{f}$ & $30^{f}$ & $422^{f}$ & $7,1^{\dagger}$ & $5,0-10,0^{f}$ & $<0,0001^{f}$ & $2,3^{f}$ & $1,6-3,4^{f}$ \\
\hline Panama $^{f}$ & $47^{f}$ & $414^{f}$ & $11,3^{f}$ & $8,6-14,8^{f}$ & $<0,0001^{f}$ & $3,9^{f}$ & $2,8-5,3^{f}$ \\
\hline Poona ${ }^{f}$ & $35^{f}$ & $396^{f}$ & $8,8^{f}$ & $6,4-12,0^{f}$ & $<0,0001^{f}$ & $2,9^{f}$ & $2,1-4,2^{f}$ \\
\hline Kentuckye & $1^{\mathrm{e}}$ & $395^{\mathrm{e}}$ & $0,3^{\mathrm{e}}$ & $0,01-1,4^{e}$ & $<0,0001^{e}$ & $0,1^{\mathrm{e}}$ & $0,01-0,6^{\mathrm{e}}$ \\
\hline Anatum ${ }^{e}$ & $3^{e}$ & $378^{e}$ & $0,8^{\mathrm{e}}$ & $0,2-2,3^{e}$ & $0,0046^{e}$ & $0,2^{\mathrm{e}}$ & $0,1-0,8^{\mathrm{e}}$ \\
\hline Brandenburg ${ }^{f}$ & $27^{\dagger}$ & $346^{4}$ & $7,8^{f}$ & $5,4-11,1^{f}$ & $<0,0001^{f}$ & $2,6^{f}$ & $1,7-3,8^{f}$ \\
\hline Derby & 5 & 342 & 1,5 & $0,6-3,4$ & 0,0868 & 0,5 & $0,2-1,1$ \\
\hline Uganda & 13 & 328 & 4,0 & $2,3-6,7$ & 0,4292 & 1,2 & $0,7-2,2$ \\
\hline Litchfielde & $2^{e}$ & $317^{e}$ & $0,6^{\mathrm{e}}$ & $0,1-2,2^{e}$ & $0,0055^{e}$ & $0,2^{e}$ & $0,1-0,8^{\mathrm{e}}$ \\
\hline Bareillye & $3^{e}$ & $308^{e}$ & $1,0^{\mathrm{e}}$ & $0,3-2,8^{e}$ & $0,0216^{e}$ & $0,3^{e}$ & $0,1-0,9 \mathrm{e}$ \\
\hline Reading ${ }^{f}$ & $28^{f}$ & $304^{f}$ & $9,2^{f}$ & $6,4-13,0^{f}$ & $<0,0001^{f}$ & $3,1^{f}$ & $2,1-4,5^{f}$ \\
\hline Hartford & 6 & 297 & 2,0 & $0,9-4,3$ & 0,3200 & 0,6 & $0,3-1,4$ \\
\hline Sandiego $f$ & $32^{f}$ & $290^{f}$ & $11,0^{f}$ & $7,9-15,2^{f}$ & $<0,0001^{f}$ & $3,7^{f}$ & $2,6-5,4^{f}$ \\
\hline Kiambu & 4 & 275 & 1,5 & $0,6-3,7$ & 0,1197 & 0,5 & $0,2-1,2$ \\
\hline Bovismorbificans & 8 & 255 & 3,1 & $1,6-6,1$ & $>0,9999$ & 1,0 & $0,5-2,0$ \\
\hline Berta $^{e}$ & $2^{e}$ & $248^{e}$ & $0,8^{\mathrm{e}}$ & $0,1-2,9^{e}$ & $0,0278^{e}$ & $0,3^{e}$ & $0,1-1,0^{\mathrm{e}}$ \\
\hline Chester $^{f}$ & $14^{f}$ & $198^{f}$ & $7,1^{f}$ & $4,3-11,5^{f}$ & $0,0066^{f}$ & $2,3^{4}$ & $1,3-4,0^{f}$ \\
\hline Dublin $^{f}$ & $66^{f}$ & $187^{f}$ & $35,3^{f}$ & $28,8-42,4^{f}$ & $<0,0001^{f}$ & $16,5^{f}$ & $12,2-22,3^{f}$ \\
\hline Corvallis & 3 & 185 & 1,6 & $0,4-4,7$ & 0,2948 & 0,5 & $0,2-1,6$ \\
\hline Manhattan & 3 & 182 & 1,6 & $0,4-4,7$ & 0,2944 & 0,5 & $0,2-1,6$ \\
\hline Mississippi & 1 & 170 & 0,6 & $0,03-3,3$ & 0,0478 & 0,2 & $0,02-1,3$ \\
\hline Tennessee & 2 & 160 & 1,3 & $0,2-4,1,4$ & 0,2534 & 0,4 & $0,1-1,5$ \\
\hline Give & 4 & 150 & 2,7 & $1,0-6,7$ & $>0,9999$ & 0,8 & $0,3-2,2$ \\
\hline Muenster & 8 & 149 & 5,4 & $2,7-10,2$ & 0,1536 & 1,7 & $0,8-3,5$ \\
\hline Hvittingfoss & 1 & 137 & 0,7 & $0,04-4,0$ & 0,1379 & 0,2 & $0,03-1,6$ \\
\hline Rissen & 2 & 134 & 1,5 & $0,3-5,3$ & 0,4533 & 0,5 & $0,1-2,1$ \\
\hline Eastbourne & 6 & 129 & 4,7 & $2,1-9,8$ & 0,3125 & 1,5 & $0,7-3,3$ \\
\hline Havana & 2 & 116 & 1,7 & $0,3-6,1$ & 0,5927 & 0,5 & $0,1-2,1$ \\
\hline
\end{tabular}


Tableau 2 : Proportions de bactériémies des sérovars de Salmonella non typhiques ayant fait l'objet d'au moins 10 signalements au PNSME, 2006 à 2019 (suite)

\begin{tabular}{|c|c|c|c|c|c|c|c|}
\hline Serovar ${ }^{a}$ & $\begin{array}{l}\text { Nombre } \\
\text { d'isolats } \\
\text { sanguins }\end{array}$ & $\begin{array}{c}\text { Nombre total } \\
\text { d'isolats }\end{array}$ & $\begin{array}{c}\text { Taux de } \\
\text { bactériémie } \\
(\%)^{\mathrm{b}}\end{array}$ & IC À 95\% (\%) & valeur-p $p^{c}$ & $\begin{array}{l}\text { Rapport des } \\
\text { cotes }^{d}\end{array}$ & IC À 95 \% \\
\hline | Rough-O::-: & 1 & 116 & 0,9 & $0,04-4,7$ & 0,1905 & 0,3 & $0,04-1,9$ \\
\hline Oslo & 2 & 106 & 1,9 & $0,3-6,6$ & 0,7781 & 0,6 & $0,1-2,4$ \\
\hline Haifa & 4 & 94 & 4,3 & $1,7-10,4$ & 0,5478 & 1,3 & $0,5-3,7$ \\
\hline London & 3 & 91 & 3,3 & $0,9-9,2$ & 0,7681 & 1,0 & $0,3-3,3$ \\
\hline Indiana & 2 & 91 & 2,2 & $0,4-7,7$ & $>0,9999$ & 0,7 & $0,2-2,8$ \\
\hline | 9,12-:- & 3 & 88 & 3,4 & $0,9-9,6$ & 0,7610 & 1,1 & $0,3-3,4$ \\
\hline Ohio & 2 & 85 & 2,4 & $0,4-8,2$ & $>0,9999$ & 0,7 & $0,2-3,0$ \\
\hline Durban ${ }^{f}$ & $8^{f}$ & $84^{f}$ & $9,5^{f}$ & $4,9-17,7^{f}$ & $0,0056^{f}$ & $3,2^{f}$ & $1,5-6,6^{f}$ \\
\hline Telelkebirf & $6^{f}$ & $75^{f}$ & $8,0^{f}$ & $3,7-16,4^{f}$ & $0,0334^{f}$ & $2,6^{f}$ & $1,1-6,1^{4}$ \\
\hline Bredeney & 4 & 71 & 5,6 & $2,2-13,6$ & 0,2921 & 1,8 & $0,7-4,9$ \\
\hline Pomona & 2 & 65 & 3,1 & $0,6-10,5$ & $>0,9999$ & 1,0 & $0,2-3,9$ \\
\hline Minnesota & 1 & 57 & 1,8 & $0,09-9,3$ & $>0,9999$ & 0,5 & $0,1-3,9$ \\
\hline Carrau & 3 & 54 & 5,6 & $1,5-15,1$ & 0,2495 & 1,8 & $0,6-5,7$ \\
\hline Cubana & 1 & 53 & 1,9 & $0,1-9,9$ & $>0,9999$ & 0,6 & $0,1-4,2$ \\
\hline Bonariensis & 4 & 52 & 7,7 & $3,0-18,2$ & 0,0487 & 2,5 & $0,9-7,0$ \\
\hline Aberdeen & 2 & 51 & 3,9 & $0,7-13,2$ & 0,6792 & 1,2 & $0,3-5,1$ \\
\hline Rubislaw & 2 & 51 & 3,9 & $0,7-13,2$ & 0,6792 & 1,2 & $0,3-5,1$ \\
\hline Gaminara & 1 & 51 & 2,0 & $0,1-10,3$ & $>0,9999$ & 0,6 & $0,1-4,4$ \\
\hline Cerro & 1 & 49 & 4,1 & $0,7-13,7$ & 0,6710 & 1,2 & $0,3-5,3$ \\
\hline Gatuni & 1 & 46 & 2,2 & $0,1-11,3$ & $>0,9999$ & 0,7 & $0,1-4,9$ \\
\hline Worthington & 1 & 45 & 2,2 & $0,1-11,6$ & $>0,9999$ & 1,0 & $0,1-5,0$ \\
\hline Urbana $^{f}$ & $4^{f}$ & $42^{f}$ & $9,5^{f}$ & $3,8-22,1^{f}$ & $0,0450^{f}$ & $3,2^{f}$ & $1,1-8,9^{f}$ \\
\hline | 6,7:r:- & 1 & 41 & 2,4 & $0,1-12,6$ & $>0,9999$ & 0,8 & $0,1-5,5$ \\
\hline Arechavaleta & 2 & 39 & 5,1 & $0,9-16,9$ & 0,3566 & 1,6 & $0,4-6,8$ \\
\hline Agbeni & 1 & 39 & 2,6 & $0,1-13,2$ & $>0,9999$ & 0,8 & $0,1-5,8$ \\
\hline Nessziona & 2 & 38 & 5,3 & $0,9-17,3$ & 0,3449 & 1,7 & $0,4-7,0$ \\
\hline Lomalinda & $5^{f}$ & $35^{f}$ & $14,3^{f}$ & $6,3-29,4^{f}$ & $0,0049^{f}$ & $5,0^{f}$ & $2,0-13,0^{f}$ \\
\hline Johannesburg & 3 & 34 & 8,8 & $3,0-23,0$ & 0,0944 & 2,9 & $0,9-9,6$ \\
\hline Choleraesuis $^{f}$ & $12^{f}$ & $33^{f}$ & $36,4^{f}$ & $22,2-53,3^{f}$ & $<0,0001^{f}$ & $17,2^{f}$ & $8,5-351^{f}$ \\
\hline Stanleyville & $7^{f}$ & $33^{f}$ & $21,2^{f}$ & $10,7-37,8^{f}$ & $<0,0001^{f}$ & $8,1^{f}$ & $3,5-18,7^{f}$ \\
\hline Ebrie & 1 & 33 & 3,0 & $0,2-15,3$ & $>0,9999$ & 1,0 & $0,1-6,9$ \\
\hline Kottbus & 1 & 32 & 3,1 & $0,2-15,7$ & $>0,9999$ & 1,0 & $0,1-7,1$ \\
\hline Livingstone & 1 & 32 & 3,1 & $0,2-15,7$ & $>0,9999$ & 1,0 & $0,1-7,1$ \\
\hline 1 6,7:c:- ${ }^{f}$ & $9^{f}$ & $30^{f}$ & $30,0^{f}$ & $16,7-47,9^{f}$ & $<0,0001^{f}$ & $12,9^{f}$ & $5,9-28,3^{f}$ \\
\hline Nima & 1 & 28 & 3,6 & $0,2-17,7$ & 0,5981 & 1,1 & $0,2-8,2$ \\
\hline Chailey & 2 & 26 & 7,7 & $1,4-24,1$ & 0,2021 & 2,5 & $0,6-10,6$ \\
\hline 16,7:k:- & 1 & 26 & 3,8 & $0,2-18,9$ & 0,5710 & 1,2 & $0,2-8,9$ \\
\hline Daytona & 1 & 25 & 4,0 & $0,2-19,5$ & 0,5569 & 1,3 & $0,2-9,3$ \\
\hline Meleagridis & 1 & 25 & 4,0 & $0,2-19,5$ & 0,5569 & 1,3 & $0,2-9,3$ \\
\hline Colindale & 1 & 24 & 4,2 & $0,2-20,2$ & 0,5422 & 1,3 & $0,2-9,7$ \\
\hline | Rough-O:g,m:- & 2 & 23 & 8,7 & $1,6-27,8$ & 0,1671 & 2,9 & $0,7-12,3$ \\
\hline Monschaui & 1 & 23 & 4,3 & $0,2-21,0$ & 0,5270 & 1,4 & $0,2-10,2$ \\
\hline Baildon & 1 & 22 & 4,5 & $0,2-21,8$ & 0,5114 & 1,4 & $0,2-10,7$ \\
\hline Coeln & 1 & 22 & 4,5 & $0,2-21,8$ & 0,5114 & 1,4 & $0,2-10,7$ \\
\hline Emek & 1 & 22 & 4,5 & $0,2-21,8$ & 0,5114 & 1,4 & $0,2-10,7$ \\
\hline Kintambo & 1 & 20 & 5,0 & $0,3-23,6$ & 0,4785 & 1,6 & $0,2-11,9$ \\
\hline Praha & 1 & 17 & 5,9 & $0,3-27,0$ & 0,4250 & 1,9 & $0,3-14,2$ \\
\hline Michigan & 1 & 16 & 6,3 & $0,3-28,3$ & 0,4060 & 2,0 & $0,3-15,2$ \\
\hline Wandsworth & 1 & 16 & 6,3 & $0,3-28,3$ & 0,4060 & 2,0 & $0,3-15,2$ \\
\hline
\end{tabular}


Tableau 2 : Proportions de bactériémies des sérovars de Salmonella non typhiques ayant fait l'objet d'au moins 10 signalements au PNSME, 2006 à 2019 (suite)

\begin{tabular}{|c|c|c|c|c|c|c|c|}
\hline Serovar ${ }^{a}$ & $\begin{array}{l}\text { Nombre } \\
\text { d'isolats } \\
\text { sanguins }\end{array}$ & $\begin{array}{l}\text { Nombre total } \\
d^{\prime} \text { 'isolats }\end{array}$ & $\begin{array}{c}\text { Taux de } \\
\text { bactériémie } \\
(\%)^{\mathrm{b}}\end{array}$ & IC À 95\% (\%) & valeur-p ${ }^{c}$ & $\begin{array}{l}\text { Rapport des } \\
\text { cotes }^{d}\end{array}$ & IC À 95 \% \\
\hline | Rough-O:r:1,2 & 1 & 15 & 6,7 & $0,3-29,8$ & 0,3864 & 2,2 & $0,3-16,4$ \\
\hline Glostrup & 1 & 14 & 7,1 & $0,4-31,5$ & 0,3661 & 2,3 & $0,3-17,7$ \\
\hline | 4,[5],12:e,h:- & 1 & 14 & 7,1 & $0,4-31,5$ & 0,3661 & 2,3 & $0,3-17,7$ \\
\hline Takoradi & 1 & 13 & 7,6 & $0,4-33,3$ & 0,3451 & 2,5 & $0,3-19,3$ \\
\hline Napoli & 1 & 12 & 8,3 & $0,4-35,4$ & 0,3234 & 2,7 & $0,4-21,3$ \\
\hline Nottingham & 1 & 10 & 10,0 & $0,5-40,4$ & $>0,9999$ & 3,4 & $0,4-26,5$ \\
\hline
\end{tabular}

Abréviations : IC, intervalle de confiance, PNSME, Programme national de surveillance des maladies entériques; SNT, Salmonella non typhique

a Les sérovars ayant fait l'objet d'au moins 10 rapports sont classés par ordre décroissant de fréquence des rapports

${ }^{b}$ Les proportions de bactériémie ont été calculées comme le nombre d'isolats sanguins par rapport au nombre total d'isolats

'Les valeurs-p ont été calculées en comparant les proportions de bactériémies de chaque sérovar avec celles du groupe de tous les sérovars non typhiques

Les rapports de cotes ont été calculés par rapport à tous les sérovars non typhiques

- Sérovars associés à des taux significativement plus faibles de bactériémie par rapport à tous les sérovars non typhiques (vert)

† Sérovars associés à des taux significativement plus élevés de bactériémie par rapport à tous les sérovars non typhiques (rouge)

Dix-neuf sérovars présentaient des taux de bactériémie significativement plus élevés que ceux du groupe de Salmonella non typhique. En termes de fréquence, ces sérovars varient du troisième le plus signalé (Heidelberg) au 85e (I 6.7:c:-). Les sérovars présentant les taux de bactériémie les plus élevés étaient Choleraesuis (33 signalements, taux de bactériémie $36,4 \%$, IC $95 \%: 22.2 \%-53,3 \%$ ) ; Dublin (187 rapports, taux de bactériémie $35,3 \%$, IC $95 \%: 28.8 \%-42,4 \%)$; et I 6,7:c:- (30 signalements, taux de bactériémie 30,0\%, IC $95 \%$ : $16.7 \%-47.9 \%)$.

Le tableau 3 énumère les sérovars de la Salmonella non typhique ayant fait l'objet d'au moins 10 signalements et n'ayant pas fait l'objet d'une isolation sanguine. Parmi ceux-ci, 20 présentaient des taux de bactériémie significativement plus faibles que le groupe de tous les sérovars de la Salmonella non typhique. Dans ce groupe se trouvaient Enteritidis et Typhimurium. En outre, 48 sérovars signalés au moins 10 fois entre 2006 et 2019 n'étaient associés à aucune isolation sanguine.

Tableau 3 : Sérovars de Salmonella signalés au PNSME en 2006 à 2019 et non associés à des bactériémies

\begin{tabular}{|l|r|r|}
\multicolumn{1}{|c|}{ Sérovar } & Total des isolats & \multicolumn{1}{c|}{ valeur-p ${ }^{a}$} \\
\hline Weltevreden & 382 & $<0,0001$ \\
\hline Senftenberg & 313 & $<0,0001$ \\
\hline Mississippi & 170 & 0,0478 \\
\hline Blockley & 117 & 0,0568 \\
\hline Miami & 105 & 0,0847 \\
\hline | 9,12:-:1,5 & 100 & 0,0804 \\
\hline Adelaide & 86 & 0,1188 \\
\hline Albany & 86 & 0,1188 \\
\hline Alachua & 54 & 0,4216 \\
\hline Concord & 53 & 0,4187 \\
\hline Cotham & 46 & 0,4059 \\
\hline Norwich & 46 & 0,4059 \\
\hline Ealing & 45 & 0,4053 \\
\hline Singapore & 44 & 0,4050 \\
\hline
\end{tabular}

Tableau 3 : Sérovars de Salmonella signalés au PNSME en 2006 à 2019 et non associés à des bactériémies (suite)

\begin{tabular}{|c|c|c|}
\hline Sérovar & Total des isolats & valeur-p ${ }^{a}$ \\
\hline Richmond & 38 & 0,6349 \\
\hline | 4,[5],12:-:1,2 & 36 & 0,6309 \\
\hline 1 6,7:-:- & 31 & 0,6260 \\
\hline Altona & 27 & $>0,9999$ \\
\hline | 4,[5],12:d:- & 27 & $>0,9999$ \\
\hline Potsdam & 25 & $>0,9999$ \\
\hline I Rough-O:HNM & 25 & $>0,9999$ \\
\hline Ago & 24 & $>0,9999$ \\
\hline Bardo & 24 & $>0,9999$ \\
\hline Irumu & 23 & $>0,9999$ \\
\hline Liverpool & 23 & $>0,9999$ \\
\hline Othmarschen & 23 & $>0,9999$ \\
\hline Istanbul & 21 & $>0,9999$ \\
\hline Fluntern & 20 & $>0,9999$ \\
\hline Putten & 20 & $>0,9999$ \\
\hline | 6,8:e,h:- & 20 & $>0,9999$ \\
\hline Kedougou & 19 & $>0,9999$ \\
\hline Larochelle & 19 & $>0,9999$ \\
\hline | 6,7:e,h:- & 19 & $>0,9999$ \\
\hline Isangi & 16 & $>0,9999$ \\
\hline Goettingen & 15 & $>0,9999$ \\
\hline Lexington & 15 & $>0,9999$ \\
\hline Amsterdam & 14 & $>0,9999$ \\
\hline Apapa & 14 & $>0,9999$ \\
\hline Kingabwa & 13 & $>0,9999$ \\
\hline | 13,23:b:- & 13 & $>0,9999$ \\
\hline Abony & 12 & $>0,9999$ \\
\hline I Rough-O:i:1,2 & 12 & $>0,9999$ \\
\hline Kisarawe & 11 & $>0,9999$ \\
\hline Saphra & 10 & $>0,9999$ \\
\hline 16,8:-:- & 10 & $>0,9999$ \\
\hline
\end{tabular}

Abréviation : PNSME, Programme national de surveillance des maladies entériques

a Les valeurs-p ont été calculées en comparant les proportions de bactériémies de chaque sérovar avec celles du groupe de tous les sérovars non typhiques 


\section{Discussion}

fréquemment signalés, Enteritidis, Typhimurium et Heidelberg, sont présentées dans la figure 7. De 2006 à 2019, le nombre de rapporte sanguins pour le sérovar Enteritidis est passé de 15 à 97 et le nombre total de signalements est passé de 1338 à 2254 , soit un taux de bactériémie qui est passé de 1,1\% (IC $95 \%$ : 0,7 \%-1,8 \%) à 4,1\% (IC $95 \%: 3,4 \%-5,0 \%, P<0.0001)$. Le taux de bactériémie de Typhimurium a également semblé augmenter au cours de la période de référence, passant de $0,4 \%$ (IC $95 \%$ : 0,2 \%-1,0 \%) à 2,3\% (IC $95 \%$ : 1,3\%-3.9\%, $P=0.0031)$. Le nombre de rapports sanguin est resté relativement stable, avec quatre en 2006 et 13 en 2019 , mais le nombre total de rapports a diminué, passant de 998 en 2006 à 557 en 2019.

Les fréquences de signalement du sérovar Heidelberg ont fluctué de manière non linéaire au cours de la période de signalement, passant d'un minimum de 19 rapports sanguins en 2019 à un maximum de 99 en 2017. Le nombre total de rapports a varié entre un minimum de 267 en 2019 et un maximum de 1071 en 2012. Le taux de bactériémie correspondant a également fluctué, passant d'un minimum de 5,4 \% (IC $95 \%$ : 3,9\%-7,5\%) en 2007 à un maximum de 18,2 \% (IC 95 \% : 15,2 \%-21,7\%) en 2017.
Selon les données soumises au Programme national de surveillance des maladies entériques, le taux global de bactériémie à Salmonella au Canada était de 4,4\% (IC $95 \%$ : $4,3 \%-4,6 \%$ ) de 2006 à 2019. Les taux annuels ont varié de $2,7 \%$ à $5,9 \%$ et ont augmenté en moyenne de $0,2 \%$ par an au cours de la période en question. L'analyse du taux de bactériémie pour les sérovars individuels a montré qu'il évoluait dans le temps en fonction du nombre de rapports totaux et sanguins. Le taux global pour le genre devrait donc refléter les changements dans les habitudes de signalement de tous les sérovars de Salmonella au Canada. Les raisons sous-jacentes de ces changements méritent d'être étudiées plus avant, mais pourraient refléter des changements dans les voies d'exposition, des changements dans la prévalence des sérovars, des changements démographiques ou autres dans la population des patients, ou une combinaison de ces facteurs $(14,15)$.

Le taux de bactériémie pour les isolats de typhoïde était de $35,2 \%$ (IC $95 \%$ : 33,7 \%-36,8 \%), au cours de la période de référence de 14 ans. La bactériémie est souvent une conséquence de la fièvre typhoïde et le taux rapporté ici est conforme aux valeurs publiées (17). Le taux de bactériémie des

Figure 7 : Taux de bactériémie des différents sérovars de Salmonella non typhique, 2006 à $2019^{a, b, c}$

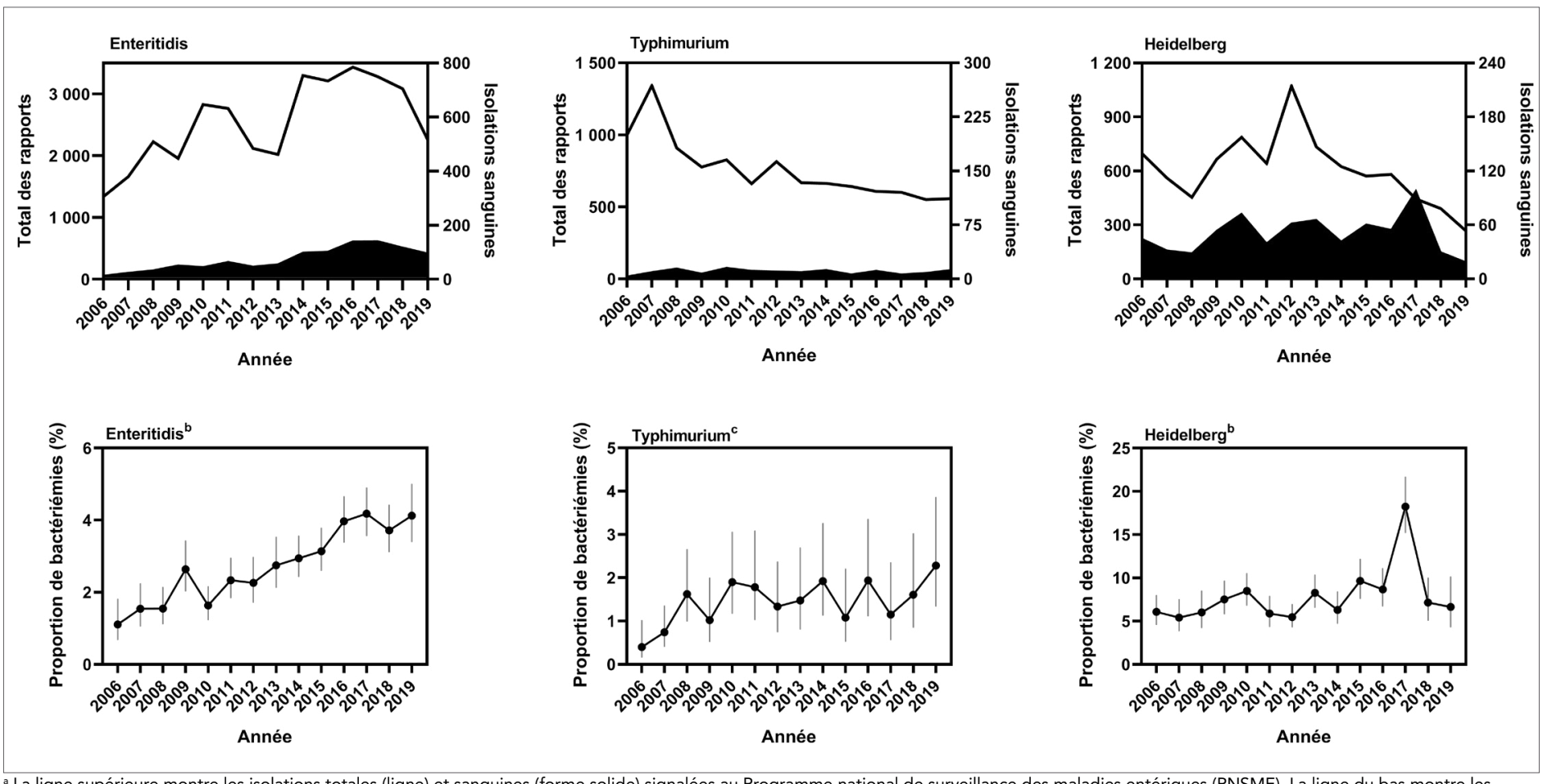

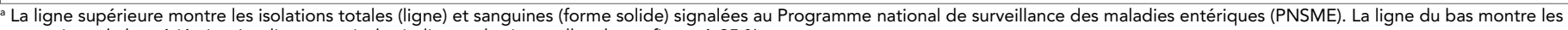
proportions de bactériémies. Les lignes verticales indiquent les intervalles de confiance à $95 \%$

b $P<0,0001$

$c P<0,0001$ 
isolats de la Salmonella non typhique était de 3,2 \% (IC $95 \%$ : $3,1 \%-3,3 \%)$. Ce taux était légèrement inférieur aux estimations des États-Unis $(5 \%)(18,19)$ et supérieur aux $2,1 \%$ rapportés pour l'Angleterre (14).

Ces différences pourraient être dues à des différences dans les périodes de signalement des trois études (États-Unis : 1996 à 2006, Angleterre : 2004 à 2015) et des différences dans la prévalence des sérovars $(14,19)$. Les cinq principaux sérovars signalés dans l'étude américaine étaient Typhimurium, Enteritidis, Newport, Heidelberg et Javiana (19). En Angleterre, les cinq principaux sérovars étaient Enteritidis, Typhimurium, Virchow, Newport et Infantis (14). On a constaté un chevauchement considérable des sérovars les plus fréquemment associés aux bactériémies au Canada, aux États-Unis et en Angleterre $(14,19)$. Parmi les 19 sérovars identifiés comme ayant des taux de bactériémie élevés dans cette étude, huit ont également été identifiés comme ayant des taux de bactériémie élevés en Angleterre et aux États-Unis : Heidelberg, Oranienburg, Virchow, Schwarzengrund, Panama, Poona, Brandenburg et Dublin. Le Canada partageait quatre sérovars supplémentaires avec les États-Unis (Reading, Sandiego, Urbana et Choleraesuis) et deux avec l'Angleterre (Chester et Paratyphi B var. Java) $(14,19)$.

Des variations géographiques des sérovars ont également été remarquées entre les trois études. Les sérovars Durban, Telelkebir, Lomalinda, Stanleyville et I 6,7:c:- ont tous été identifiés dans cette étude comme ayant des taux de bactériémie plus élevés. Collectivement, ces cinq sérovars sont rares et ont été à l'origine de 257 cas confirmés en laboratoire entre 2006 et 2019 (soit 0,3\% de l'ensemble des signalements de Salmonella non typhique). Ces sérovars n'étaient pas répertoriés dans les rapports anglais et américains, ce qui suggère que les expositions sont liées à des voyages ou sont uniques au Canada.

La poursuite de l'étude de la pathogenèse des sérovars de la Salmonella non typhique associés et non associés à la bactériémie permettra d'acquérir des connaissances sur les risques relatifs des sérovars de Salmonella. La compréhension de ces différences permettra d'élaborer des méthodes pour améliorer les soins aux patients grâce à l'identification et au traitement précoces des cas de salmonellose qui présentent un risque élevé de développer une bactériémie.

\section{Forces et faiblesses}

Les taux de bactériémies présentés ci-dessus sont des estimations du taux réel fondées sur les données soumises au Programme national de surveillance des maladies entériques. La soumission des données au Programme national de surveillance des maladies entériques est volontaire. Les valeurs indiquées dans ce rapport ne représentent que les isolats soumis par les laboratoires provinciaux et territoriaux et sous-estiment le nombre de cas de salmonellose au Canada (20).
Les données sur la source de l'isolat sont saisies par le Programme national de surveillance des maladies entériques sur la base des détails recueillis par les laboratoires soumissionnaires. Comme cette information n'est pas toujours disponible, les données sur les infections extra-intestinales à Salmonella peuvent également être sous-déclarées. Bien que tous les efforts soient faits pour éviter les doublons, les données sources limitées ne permettent pas d'identifier les isolations en double provenant de différentes sources et provenant du même patient. De plus, les données incluent les cas liés aux voyages, ce qui ne reflète pas la véritable représentation des souches endémiques au Canada.

La solidité d'études comme celle-ci repose sur la qualité et la quantité des données de surveillance disponibles. Un nombre plus faible d'isolats rapportés conduit à un plus grand degré d'imprécision. Les chiffres présentés ici sont probablement biaisés en faveur de la bactériémie, car les patients présentant des symptômes plus graves sont plus susceptibles de consulter un médecin que ceux qui présentent une forme autolimitée de la maladie. En l'absence de données cliniques, on suppose que les cas dans lesquels des isolats sanguins de Salmonella ont été récupérés étaient plus graves que ceux qui avaient des cultures de selles positives.

\section{Conclusion}

D'après les données soumises au Programme national de surveillance des maladies entériques, on estime que 4,4\% des cas de salmonellose survenus entre 2006 et 2019 ont entrainé une bactériémie. Des groupes et sérovars spécifiques de Salmonella associés à des taux de bactériémie plus ou moins élevés par rapport au groupe plus large de souches de Salmonella ont été identifiés. Les résultats de cette étude éclaireront les recherches futures visant à prédire et à améliorer l'issue des bactériémies à Salmonella au Canada.

\section{Déclaration des auteurs}

S. T. - Conceptualisation, méthodologie, analyse formelle, rédaction-version originale, rédaction-révision et édition B. D. - Rédaction-révision et édition

K. N. - Curation des données, rédaction-révision et édition

\section{Intérêts concurrents}

Aucun.

\section{Remerciements}

Les auteurs tiennent à remercier le personnel de l'Agence de la santé publique du Canada, du Programme des maladies entériques du Laboratoire national de microbiologie et 
du Centre des maladies infectieuses d'origine alimentaire, environnementale et zoonotique qui administre le Programme national de surveillance des maladies entériques. Les auteurs remercient également à $\mathrm{A}$. Catford, E. Buenaventura, B. Dixon et J. Austin pour la révision critique du manuscrit.

\section{Financement}

Ce travail a été financé par Santé Canada (services votés) pour soutenir les programmes de sécurité alimentaire du Canada.

\section{Références}

1. Jajere SM. A review of Salmonella enterica with particular focus on the pathogenicity and virulence factors, host specificity and antimicrobial resistance including multidrug resistance. Vet World 2019;12(4):504-21. DOI PubMed

2. Lamas A, Miranda JM, Regal P, Vázquez B, Franco CM, Cepeda A. A comprehensive review of non-enterica subspecies of Salmonella enterica. Microbiol Res 2018;206:60-73. DOI PubMed

3. Gal-Mor O, Boyle EC, Grassl GA. Same species, different diseases: how and why typhoidal and non-typhoidal Salmonella enterica serovars differ. Front Microbiol 2014;5:391. DOI PubMed

4. Thomas MK, Murray R, Flockhart L, Pintar K, Fazil A, Nesbitt A, Marshall B, Tataryn J, Pollari F. Estimates of foodborne illness-related hospitalizations and deaths in Canada for 30 specified pathogens and unspecified agents. Foodborne Pathog Dis 2015;12(10):820-7. DOI PubMed

5. Ilyas B, Tsai CN, Coombes BK. Evolution of Salmonella-host cell interactions through a dynamic bacterial genome. Front Cell Infect Microbiol 2017;7:428. DOI PubMed

6. Agence de la santé publique du Canada. Programme national de surveillance des maladies entériques. 2018 (accédé 2020-12-04). https://www.canada.ca/fr/santepublique/programmes/programme-national-surveillancemaladies-enteriques.html

7. Cheng RA, Eade CR, Wiedmann M. Embracing diversity: differences in virulence mechanisms, disease severity, and host adaptations contribute to the success of nontyphoidal Salmonella as a foodborne pathogen. Front Microbiol 2019;10:1368. DOI PubMed

8. Gal-Mor O. Persistent infection and long-term carriage of typhoidal and nontyphoidal salmonellae. Clin Microbiol Rev 2018;32(1):e00088-18. DOI PubMed
9. Hohmann EL. Nontyphoidal salmonellosis. Clin Infect Dis 2001;32(2):263-9. DOI PubMed

10. Matheson N, Kingsley RA, Sturgess K, Aliyu SH, Wain J, Dougan G, Cooke FJ. Ten years experience of Salmonella infections in Cambridge, UK. J Infect 2010;60(1):21-5. DOI PubMed

11. Robinson JL. Salmonella infections in Canadian children. Paediatr Child Health 2019;24(1):50-1. DOI PubMed

12. Galanakis E, Bitsori M, Maraki S, Giannakopoulou C, Samonis G, Tselentis Y. Invasive non-typhoidal salmonellosis in immunocompetent infants and children. Int J Infect Dis 2007;11(1):36-9. DOI PubMed

13. Hsu RB, Tsay YG, Chen RJ, Chu SH. Risk factors for primary bacteremia and endovascular infection in patients without acquired immunodeficiency syndrome who have nontyphoid salmonellosis. Clin Infect Dis 2003;36(7):829-34. DOI PubMed

14. Katiyo S, Muller-Pebody B, Minaji M, Powell D, Johnson AP, De Pinna E, Day M, Harris R, Godbole G. Epidemiology and outcomes of nontyphoidal Salmonella bacteremias from England, 2004 to 2015. J Clin Microbiol 2019;57(1):e01189-18. DOI PubMed

15. GBD 2017 Non-Typhoidal Salmonella Invasive Disease Collaborators. The global burden of non-typhoidal salmonella invasive disease: a systematic analysis for the Global Burden of Disease Study 2017. Lancet Infect Dis 2019;19(12):1312-24. DOI PubMed

16. Mandal BK, Brennand J. Bacteraemia in salmonellosis: a 15 year retrospective study from a regional infectious diseases unit. BMJ 1988;297(6658):1242-3. DOI PubMed

17. Wain J, Diep TS, Ho VA, Walsh AM, Nguyen TT, Parry CM, White NJ. Quantitation of bacteria in blood of typhoid fever patients and relationship between counts and clinical features, transmissibility, and antibiotic resistance. J Clin Microbiol 1998;36(6):1683-7. DOI PubMed

18. Arshad MM, Wilkins MJ, Downes FP, Rahbar MH, Erskine RJ, Boulton ML, Younus M, Saeed AM. Epidemiologic attributes of invasive non-typhoidal Salmonella infections in Michigan, 1995--2001. Int J Infect Dis 2008;12(2):176-82. DOI PubMed

19. Jones TF, Ingram LA, Cieslak PR, Vugia DJ, Tobin-D'Angelo M, Hurd S, Medus C, Cronquist A, Angulo FJ. Salmonellosis outcomes differ substantially by serotype. J Infect Dis 2008;198(1):109-14. DOI PubMed

20. Thomas MK, Murray R, Flockhart L, Pintar K, Pollari F, Fazil A, Nesbitt A, Marshall B. Estimates of the burden of foodborne illness in Canada for 30 specified pathogens and unspecified agents, circa 2006. Foodborne Pathog Dis 2013;10(7):639-48. DOI PubMed 